\title{
Within-generation and transgenerational social plasticity interact during rapid adaptive evolution
}

\author{
Samantha L. Sturiale ${ }^{1,2}$, Nathan W. Bailey ${ }^{1}$ \\ ${ }^{1}$ School of Biology, University of St Andrews, St Andrews, Fife KY16 9TH, UK \\ 2 Current address: Department of Biology, Georgetown University, Washington DC 20057, USA
}

\section{Correspondence}

Samantha L. Sturiale

Email: sls366@georgetown.edu

Nathan W. Bailey

Email: nwb3@st-andrews.ac.uk

\section{Conflicts of Interest}

We declare that we have no conflicts of interest.

\section{Author Contributions}

SLS and NWB designed experiments; SLS conducted experiments and analyzed the data; SLS wrote the first draft of the manuscript; NWB provided feedback and made conceptual contributions to the following drafts. All authors approved the final manuscript and agreed to be held accountable for its content.

\section{Data Availability Statement}

All data underlying the manuscript will be made publicly available in an appropriate repository (e.g., Dryad) upon acceptance for publication.

\section{Acknowledgements}

We are grateful for support from the Natural Environment Research Council to NWB (NE/L011255/1 and NE/T000619/1). T. Hitchcock patiently tolerated the execution of a cricket experiment in a converted spare room of his flat during the first 2020 UK Covid-19 lockdown. We are grateful for the assistance of D. Forbes, A. Grant and M. McGunnigle in cricket rearing and laboratory maintenance. S. Pascoal advised on technical aspects of the social environment manipulation. J.G. Rayner provided valuable feedback that improved the 
bioRxiv preprint doi: https://doi.org/10.1101/2021.10.21.464843; this version posted October 22, 2021. The copyright holder for this preprint (which was not certified by peer review) is the author/funder, who has granted bioRxiv a license to display the preprint in perpetuity. It is made available under aCC-BY-NC-ND 4.0 International license.

experimental design, and T.M. Jones and L.E. Rendell gave helpful input on the writing and interpretation of results. 


\section{Abstract}

1. The role of within-generation phenotypic plasticity (WGP) versus transgenerational plasticity (TGP) during evolutionary adaptation are not well understood, particularly for socially-cued TGP.

2. We tested how genetics, WGP, and TGP jointly influence expression of fitness traits facilitating adaptive evolution in the field cricket Teleogryllus oceanicus. A malesilencing mutation (“flatwing”) spread to fixation in ca. 50 generations in a Hawaiian cricket population attacked by acoustically-orienting parasitoids. This rapid loss of song caused the social environment to dramatically change.

3. Juveniles carrying the flatwing $(f w)$ genotype exhibited greater locomotive activity than those carrying the normal-wing ( $n w$ ) allele, consistent with genetic coupling of increased locomotion with $f w$.

4. Consistent with adaptive WGP, homozygous $f w$ females developing in the absence of song showed reduced body condition and reproductive investment at adulthood.

5. Adult but not juvenile offspring exhibited TGP in response to maternal social environment for structural size, somatic condition, and reproductive investment, whereas adult locomotion and flight was only influenced by WGP. WGP and TGP interacted to shape multiple traits at adulthood, though effect sizes were modest.

6. Interactions between genetic effects and social plasticity within and across generations are likely to have influenced the evolutionary spread of flatwing crickets. However, interactions among these effects can be complex, and it is notable that TGP manifested most strongly later in development. Our findings stress the importance of evaluating trait plasticity at different developmental stages and across generations when studying phenotypic plasticity's role in evolution.

Key words: adaptive evolution, maternal effects, open field test, phenotypic plasticity, pleiotropy, social environment, transgenerational plasticity 


\section{Introduction}

27 Dissecting how phenotypic plasticity affects trait expression within and across generations is necessary to fully understand its role in adaptive evolution. Within-generation plasticity (WGP), where an individual's phenotype shifts as a response to its own environmental conditions, has long been argued to influence evolutionary processes (Robinson and Dukas 1999; Huey et al. 2003; West-Eberhard 2003; Ghalambor et al. 2007; Lande 2009; Chevin et al. 2010). More recently, researchers have explored plastic responses to parental or grandparental environmental conditions through transgenerational plasticity (TGP) (LaMontagne and McCauley 2001; Dyer et al. 2010; Sheriff et al. 2010). It is clear from this work that TGP can also modify a species' evolutionary trajectory. In particular, both WGP and TGP may mitigate indirect fitness costs caused by new genetic variants under selection (negative pleiotropy), thus facilitating spread and fixation of de novo adaptive variants. However, few empirical studies of rapid adaptation have considered the consequences of both these forms of plasticity acting simultaneously.

Depending on the nature of the interaction between WGP and TGP, various evolutionary outcomes could occur. For example, a study of anti-predator defensive helmet formation in Daphnia cucullate found that WGP and TGP additively contribute to offspring phenotype (Agrawal et al. 1999). Such a relationship could move a population towards a new trait optimum faster than if just one form of plasticity were acting (Auge et al. 2017). Several other studies have detected a more complicated pattern, where parental environment interacts with the effects of offspring environment (i.e., TGP alters the extent and/or direction of offspring WGP response or reaction norm) (Prasad et al. 2003; Donelan and Trussell 2015; Luquet and Tariel 2016; Stein et al. 2018; Zirbel et al. 2018). This interaction could be adaptive, for example, by allowing parents to produce pre-adapted offspring that do not need to themselves express costly plasticity (Luquet and Tariel 2016). Alternatively in cases where TGP is non-adaptive for offspring, WGP acting on offspring traits in the opposite direction as TGP could allow offspring a means of 'escaping' negative fitness effects carried over from parental environment (Auge et al. 2017). The relationship between TGP and WGP has been somewhat explored in response to the environmental effects of predation (Agrawal et al. 1999; Donelan and Trussell 2015; Luquet and Tariel 2016; Stein et al. 2018), nutrition (Prasad et 
57 interaction in other important contexts, such as the social environment, remains largely

58 unknown.

To experimentally dissect this interaction, we tested phenotypic and fitness effects of maternal and offspring social environment in a rapidly-evolving population of the Oceanic field cricket, Teleogryllus oceanicus. In Hawaii, acoustically signaling males are attacked by an acoustically orienting parasitoid fly, Ormia ochracea. Recently, a single-locus, X-linked mutation, flatwing $(f w)$, arose and spread in fewer than 20 generations to affect ca. $90 \%$ of males in a population on the island of Kauai (Zuk et al. 2006). Fw segregates as a single-locus mutation and disrupts normal wing development, thus silencing 'flatwing' males and shielding them from fly attack (Zuk et al. 2006; Pascoal et al. 2020). Its rapid spread to fixation dramatically changed the social environment by eliminating the conspicuous longrange male acoustic signal that functions in mate attraction, courtship, and intrasexual aggression. Thus, the mutation has obvious negative indirect (pleiotropic) effects: flatwing males cannot acoustically advertise for mates. They also show partial, apparently maladaptive feminization of phenotypes unrelated to wings (Bailey et al. 2010; Pascoal et al. 2016; Pascoal et al. 2018; Rayner et al. 2019b; Pascoal et al. 2020). Pre-existing WGP to acoustic cues in the environment that offsets some of these fitness costs may have facilitated the rapid spread of flatwing. When raised in an environment lacking song, females relax mate preferences and increase responsiveness to calling males, likely enabling them to locate the few remaining singing males, or satellite flatwing males near them, in flatwingdominated populations (Bailey and Zuk 2008; 2012). Similarly, males raised in silence are more likely to express satellite mating tactics (Bailey et al. 2010). Males also show an overall increase in locomotive behavior when reared in silence (Balenger and Zuk 2015). Finally, when exposed to song during rearing, individuals of both sexes develop increased reproductive tissue mass and have enhanced immune responses compared to their counterparts raised in the absence of song (Bailey et al. 2010, 2011; Lierheimer and Tinghitella 2017; Heinen-Kay et al. 2019).

Several features of this field cricket system suggest that socially-induced TGP could also contribute to the rapid spread of flatwing males. Like many insect species, T. oceanicus suffers high juvenile mortality, favoring fitness-increasing alterations to early juvenile

87 phenotype via TGP. Recent findings suggest that nongenetic inheritance can affect the 88 interplay between predation risk vs. movement towards singing males, though this has only 
been examined for adults (Moschilla et al. 2021). Second, the fragmented distribution of $T$. oceanicus habitat in Hawaii, the inability of juveniles to fly, and overlapping generations should result in high autocorrelation between parental and offspring social environments, which is predicted to favor adaptive TGP (Leimar and McNamara 2015). Third, crickets do not possess a fully-developed auditory system until adulthood, though late juveniles may have limited auditory capabilities (Young and Ball 1974; Yack 2004; Staudacher 2009). Young juveniles are therefore not likely capable of accurately assessing their own social environment acoustically, which theory predicts will favor the evolution of TGP (Leimar and McNamara 2015).

We performed three experiments to dissect the potential contributions and interactions of genetic evolutionary responses, WGP, and TGP to rapid adaptation observed in Hawaiian T. oceanicus. Supplementary figure S1 provides an experimental overview. Across these experiments, we focused on four traits relevant to mate competition and the loss of acoustic sexual signaling during the evolutionary spread of flatwing crickets: structural size (in juveniles and adults), body condition (in adults), investment in reproductive tissues (in adults), and locomotive activity (in juveniles and adults). Locomotion was a key trait because there is evidence from other insect species that individuals exposed to crowded conditions increase dispersal themselves (WGP) or produce offspring with increased dispersal tendencies (TGP) (Denno and Roderick 1992; Allen et al. 2008; Yu et al. 2019). Locomotive activity is also expected to have especially important fitness consequences in the cricket system given the challenges of locating conspecifics in a song-less, flatwing-dominated population.

In Experiment 1, we measured how juvenile locomotive behavior varied across $f w$ and normal-wing ( $n w$ ) genotypes, before any maternal social manipulation. This allowed us to determine whether the rapid spread of flatwing males might have been associated with

114 genetic changes in juvenile expression of a trait relevant to the changing social environment.

115 We expected that fw-carriers benefit from dispersing less as juveniles and therefore aggregating at higher densities upon reproductive maturity, increasing the chances of mating

117 in a song-less environment. In Experiment 2, we investigated WGP to the acoustic social 118 environment. We focused on $f w$-carrying individuals because the source population is now 119 all-flatwing (Tinghitella et al. 2018; Rayner et al. 2019a) and previous work indicated that 120 the mutation is associated with increased socially-induced WGP (Pascoal et al. 2018). We 
121 tested whether, consistent with previous studies on females carrying $n w$ genotypes, fw-

122 carrying females (the maternal generation) raised in different acoustic environments

123 increase reproductive investment and alter mating behaviors in a way that increases 124 probability of mating with a silent male. Finally, in Experiment 3, we tested

125 transgenerational consequences of the maternal social environment by measuring size and 126 locomotive activity in acoustically-naïve juveniles, and final size, somatic condition, 127 reproductive investment, and locomotive activity in adult offspring. In this last experiment, 128 adults were exposed to either matched or mis-matched acoustic cues compared to their mothers, permitting a direct test of the interaction between WGP and TGP.

Methods

\section{Experiment 1: Genotypic differences in juvenile locomotion}

\section{Cricket populations and rearing}

133 We compared early juvenile behavior across the two cricket morph genotypes using 6 134 laboratory stock lines -3 pure-breeding for $f w$ and 3 pure-breeding for $n w$. Lines were established in 2016 from a series of controlled crosses of Kauai-derived individuals to ensure homozygosity (Pascoal et al. 2016). Stock crickets were kept in 16 litre plastic containers with

137 cardboard egg cartons for shelter. Twice weekly, they were provided ad libitum food (Burgess

138 Supa Rabbit Exel Junior pellets; blended for juveniles) and moistened cotton for water and oviposition. Crickets in isolated-rearing conditions were kept in $100 \mathrm{~mL}$ plastic deli pots with shelter, food, and water as above. All subjects were kept in the same growth chamber at $25^{\circ} \mathrm{C}$

142 juveniles for this experiment, we collected eggs from each line twice weekly for 4 weeks. After 143 approximately two weeks we monitored egg pads daily (16:00 - 18:00) and isolated new 144 hatchlings.

\section{Open-field test}

146 An open field test (OFT) was used to track individual crickets' movements in an unobstructed 147 arena and measure their total distance travelled, a useful proxy for measuring behaviors 148 related to dispersal, mate location and foraging (Fraser et al. 2001; Dingemanse et al. 2003; 149 Korsten et al. 2013). For this experiment, juveniles were isolated at hatching, and each was 
tested in an OFT at 15-days and 45 days post-hatching. Juveniles of these ages do not have mature hearing structures (Young and Ball 1974). All OFTs were performed under red light during the dark portion of the crickets' $12: 12$ light:dark cycle, between $23-25^{\circ} \mathrm{C}$. Subjects were placed in small glass vials within their deli pot to reduce handling disturbance before testing. The vial was gently turned over onto the center of an $11 \times 17 \mathrm{~cm}$ clear plastic arena atop white poster paper and the cricket was allowed to acclimatize for two minutes. Upon lifting the vial, we began recording for 5 minutes at 30 frames/second using a camera (Nikon D3300) mounted ca. $40 \mathrm{~cm}$ above the arena. The arena was wiped down with $70 \%$ ethanol before each trial to minimize residual chemical cues. Two crickets were assayed at once in side-by-side arenas. It is unlikely that they were aware of one another due to their inability to see in red wavelengths of light. After the OFT, each cricket was photographed overtop a micrometer using a Leica DFC295 digital camera affixed to a Leica M60 dissecting microscope. ImageJ (v.1.8.0_112) was used to record pronotum length (a proxy for structural size) from the images.

\section{Locomotion measurements}

We used DORIS v.0.0.17 (Friard 2019) to extract coordinates of the test subject within each video frame, followed by coordinate path smoothing implemented in $\mathrm{R}$ ( $\mathrm{R}$ Core Team 2020) to increase measurement precision (see Supplemental Methods and Figure S2). Using these coordinates, we measured total distance traveled ("distance") during trials. In this and later experiments involving open field tests, we also explored other movement parameters ("proportion explored" as a measure of exploratory activity; and "origin time", "middle time", and "edge time" as measures of space usage and thigmotaxis). However, variation in these parameters was largely accounted for by overall differences in distance moved, confirming that distance was the most salient locomotion trait in the experiment. For completeness, we discuss the measurement and analysis of all other movement traits in the Online Supplementary Information.

\section{Statistical analyzes}

All statistical tests were carried out using $R$ version 4.0.2 (R Core Team 2020). We compared distance between wing morph genotypes in 15-day old and 45-day old offspring using a linear model. Individuals who jumped during their assay ( $\mathrm{n}=2$ in 45 -day assay) or whose video was 
inadvertently deleted before analysis ( $\mathrm{n}=2$ in 45-day assay) were excluded. All data transformations are shown in Table S1.

Morph and sex were modelled as categorical variables, with line nested within morph to account for inter-line variation. Pronotum length, temperature, and time of day were included as covariates. Thirty-nine individuals died before their sex could be identified, so to verify that sex did not qualitatively affect the findings, models including sex as a fixed effect were run on the subset of individuals for which sex could be identified. Sex did not approach significance in this model (all $p>0.2$ ) and the qualitative outcome did not differ. Thus, the model retaining all individuals, and excluding sex as a fixed effect, was retained (Equation 1 of Supplementary Table S2). Finally, the model was run first with all individuals, then with only those who moved during the assay to confirm that genotypic variation in distance was not due to differences in the likelihood of initiating movement. Excluding crickets that failed to initiate movement did not affect interpretations of genotype differences, so final models included stationary crickets (Supplementary Table S3).

\section{Experiment 2: Social plasticity in the maternal generation (WGP)}

\section{Cricket populations and rearing}

The three pure-breeding $f w$ lines used in Experiment 1 were reciprocally interbred to create an admixed pure-breeding $f w$ stock population. Following previous work, we isolated juvenile females from this stock when sex became apparent to ensure virginity and more easily manipulate their acoustic environment (Bailey and Zuk 2008; Pascoal et al. 2018). We also segregated a group of juvenile males into single-sex 16-L box to maintain their virginity. All group rearing conditions were identical to Experiment 1. Isolated females were placed in a separate, temperature-controlled $25^{\circ} \mathrm{C}$ incubator on a $12 \mathrm{~h}: 12 \mathrm{~h}$ photo-reversed light cycle, with no male calling. Females were checked daily for adult eclosion, whereupon they were haphazardly assigned one of two acoustic social treatments: Song or No Song. Females do not achieve reproductive maturity until several days after adult eclosion, so our acoustic treatment targets the developmental period when mate assessment is possible but mating is not (Swanger and Zuk 2015). We also recorded the number of days spent isolated prior to eclosion to account for any differences in growth rate that might be associated with time spent without song prior to adult acoustic treatment. We kept each female in their acoustic treatment for 15 days post-eclosion. 
212 In the Song treatment, Kauai male calls reflecting population averages for key song 213 parameters were played at 80-85 dB (measured at the lid of the deli cup which has an acoustic 214 impedance of ca. $10 \mathrm{~dB}$ ) during the night portion of the crickets' light:dark cycle to best match 215 calling dynamics in the wild (Zuk et al. 1993). Playbacks used in the Song treatment have 216 been previously described (Pascoal et al. 2018) (see Supplementary Methods). Acoustic 217 treatments were run in two separate LMS Series 4 (Model 600) controlled temperature 218 incubators at $25^{\circ} \mathrm{C}$ on the same $12 \mathrm{~h}: 12 \mathrm{~h}$ photo-reversed light:dark cycle as the general 219 incubator. Calls were broadcast from computer speakers (Logitech Z120 2.0) and the calling 220 schedule programmed using the Task Scheduler application on a desktop computer. Twice a 221 week, we switched which incubator housed each acoustic treatment to prevent any incubator222 related experimental confounds.

224 At 15 days post-eclosion, isolated adult females were weighed and their pronotum width was measured using digital calipers. Each female was placed in a 16 x $18 \mathrm{~cm}$ plastic container with cardboard, rabbit chow, and moistened cotton. We haphazardly selected an adult virgin male from the flatwing stock population, weighed it, measured its pronotum width, and placed it in the container with the female. Trials were performed between $20-23^{\circ} \mathrm{C}$ under red light between 16:00h and 18:00h. They lasted for 20 minutes, and we noted whether the

230 female mounted the male and whether the male transferred a spermatophore. Afterwards, 231 pairs were placed in a separate incubator without male song at the same temperature and 232 light:dark cycle as in Experiment 1. After 24 hours, the male was removed to reduce potential 233 paternal influences on offspring phenotype. After another 24-48 hours, the female was 234 removed, and the egg pad was collected for use in Experiment 3.

To compare female body condition, we used pronotum width and total body weight to 237 calculate the scaled mass index (SMI) of each individual (Peig and Green 2009). A subset of 238 females drawn haphazardly from each treatment (total $n=23$ ) were dissected at 15 days post-eclosion rather than mated. We recorded their pronotum width using digital calipers, 
weighed them, and then determined wet mass of their dissected ovaries. Somatic mass was calculated by subtracting ovary mass from total weight.

\section{Statistical analyzes}

First, we compared $S M I$ across acoustic treatments using a linear model (Equation 2 of Table S2) with acoustic treatment as a categorical factor, days isolated before treatment as a covariate, and experimental replicate (block one or block two of the experiment). Replicate only had two factor levels so we included it as a fixed effect. Second, we compared mating behavior across acoustic treatments. We first ran a generalized linear model (GLM) with binomial error to examine presence vs. absence of female mounting during trials, including acoustic treatment, female SMI, and male SMI as predictors. Next, we ran a binomial GLM examining presence vs. absence of spermatophore transfer. For this, we only included the 49 mating trials (out of 65 total) where mounting had occurred, because spermatophore transfer cannot occur without mounting. Acoustic treatment was included as a categorical factor and female SMI and male SMI were included as covariates. Equation 3 in Table S2 gives the general form of these models.

Finally, we compared female reproductive investment (ovary mass) across acoustic treatments in the subset $(n=23)$ of females that had been dissected by running a linear model (LM) on ovary mass, with acoustic treatment and days isolated as predictor and covariate, respectively. As in previous studies of reproductive investment, we controlled for body size by including log-transformed soma mass as an additional covariate (Tomkins and Simmons 2002; Bailey et al. 2010) (Equation 4 in Table S2).

Experiment 3: Transgenerational effects of maternal social environment and interactions between TGP and WGP in adult offspring

\section{Cricket populations and rearing}

Eggs produced by the maternal generation in Experiment 2 were first kept in a separate incubator under the same temperature and light conditions as the general incubator. As they began to hatch, the first UK national lockdown in response to the 2020 Covid-19 pandemic (23 March 2020) required that all laboratory experiments be run under strict social distancing measures, which affected where and how some of our procedures were executed. 
269

270

271

272

273

274

275

276

277

278

279

280

281

282

283

284

285

286

287

288

289

290

291

292

293

294

295

296

297

298

A description of 'socially-distanced' methods plus our design to statistically account for any variation it introduced is provided in the Supplementary Methods.

We first tested TGP effects in juvenile offspring. Hatchlings were isolated as described previously and kept at $18-24^{\circ} \mathrm{C}$ on a $12 \mathrm{~h}: 12 \mathrm{~h}$ photo-reversed light:dark cycle. We ran the experiment in two blocks, with individuals in the second block kept at $25^{\circ} \mathrm{C}$ in the lab incubator, as in Experiment 1. For the early juvenile offspring TGP experiment, we tested 311 offspring (131 from 14 mothers treated with Song, 180 from 21 mothers treated with No Song) at 15 days post-hatching. 199 individuals were tested again at 45 days post-hatching (117 from 11 No Song mothers and 82 from 8 Song mothers).

For adult offspring experiments, hatchlings were kept in 10 replicate group-rearing boxes during development to match the demographic rearing conditions experienced by the previous generation. Once sex was apparent during development, individuals were isolated and assigned to either acoustic treatment using the same incubators and playback schedules as in Experiment 2. The distribution of adult offspring $(\mathrm{n}=387)$ across the 4 maternaloffspring acoustic treatment combinations is shown in Supplementary Table S4. It is important to note that the adults measured in this experiment were not the same individuals as those used for the TGP juvenile trials described above; thus, the corresponding data sets are derived from different individuals of the same generation.

Open field test

OFT procedures were identical to Experiment 1. For juvenile offspring, OFTs were performed 15 days post-hatching and 45 days post-hatching. Adult offspring OFTs were performed 8days post-adult eclosion. All recordings were performed at $23-28^{\circ} \mathrm{C}$ between 12:00 and 17:00 under dim red lighting. Adult OFTs were identical to those of juveniles, except a larger plastic arena was used (41 cm wide, $37 \mathrm{~cm}$ long, and $28 \mathrm{~cm}$ high). In the course of the experiment, we noticed that some adults attempted to fly out of the arena during the assay. When that happened, we stopped the recording and placed the subject into an incubator without song for 10 minutes. After re-acclimation, we started the trial again. The number of flight attempts was recorded for each individual. We collected movement coordinates and calculated distance using DORIS (v.0.0.17) as in Experiment 1.

Morphological measurements

Page $\mathbf{1 0}$ of $\mathbf{3 0}$ 
Following the OFT, we photographed each juvenile overtop a micrometer using the same camera and dissecting scope as in Experiment 1 and measured pronotum length using ImageJ (v.1.8.0_112). We euthanized adults after OFTs at 8 days post-eclosion, then weighed them and measured pronotum length to the nearest $0.01 \mathrm{~mm}$ using digital calipers. We then dissected, blotted excess fluid, and weighed their gonads (male testes and accessory glands, female ovaries). Here we used pronotum length and soma weight to calculate SMI, by subtracting gonad weight from total weight. In this experiment, SMI was thus a measure of somatic body condition, which allowed us to investigate whether differences in maternal or offspring acoustic environments influenced relative investment in somatic tissues while scaling to structural size. SMI was calculated separately for each sex. Gonad weight was later compared directly.

\section{Statistical analyzes}

First, we tested whether juvenile offspring size differed between maternal acoustic treatments by running a linear mixed model (LMM) using pronotum length as the response. Maternal treatment and experimental replicate were included as fixed effects, with maternal ID as a random effect. Experimental replicate was included to account for different rearing temperatures in trial 1 and trial 2 (see Supplementary Methods). The treatment*replicate interaction $(p>0.2)$ was excluded from the final model. Pronotum length of 45-day old offspring was modelled similarly except experimental replicate was not included because we only had 45-day nymph data for trial 1. Models took the general form of Equation 5 in Supplementary Table S2.

We then examined the effect of maternal treatment on juvenile offspring distance using an LLM which included maternal treatment and experimental replicate as categorical factors; temperature, time of day, and pronotum length as covariates; and maternal ID as a random effect. The treatment*replicate interaction $(p>0.2)$ was excluded from the final model. A similar model was run for 45-day old offspring, except experimental replicate was not included because we only had 45-day data for trial 1 . The general form of the model is given by Equation 6 in Supplementary Table S2.

To investigate TGP and WGP and their interaction, we tested the effect of maternal and offspring acoustic treatments on adult pronotum length and somatic condition (SMI). Because there are large sex differences in physiology and the possibility of sex-specific 
maternal effects, we ran separate models for each sex. Each LMM included maternal and offspring treatments as factors plus their interaction. Non-significant $(p>0.2)$ interactions were removed. We analyzed the effect of acoustic treatments on adult offspring reproductive investment using sex-specific models with gonad weight as the response. First we compared unscaled reproductive investment, then we added pronotum length as a covariate to examine whether variation in reproductive investment could be explained by structural size. Finally,

336 we added log-transformed somatic mass to examine whether variation in reproductive 337 investment might be explained by somatic weight. Replicate was included as a random effect. 338 These models took the general form shown in Equation 7 of Supplementary Table S2.

We then tested the impact of TGP and WGP on adult distance using separate LMMs for each behavior and sex. All models included maternal treatment, offspring treatment, 341 temperature, time, and somatic SMI, plus replicate as a random effect (Equation 8 in Table S2). Non-significant $(\mathrm{p}>0.2)$ maternal*offspring treatment interactions were removed.

As a post hoc analysis of treatment and sex variation in attempted flight behavior, we 344 modelled flight attempts using a generalized linear mixed model (GLMM) with a binomial 345 error (1 if flight was attempted and 0 if not) with the predictors: maternal treatment, 346 offspring treatment, sex, maternal treatment*sex, and somatic SMI. Replicate was included 347 as a random effect. Equation 9 of Supplementary Table S2 describes the final model after removing non-significant interaction terms. 


\section{Results}

350 Experiment 1: Genotypic differences in juvenile locomotion

$F w$-carrying nymphs moved further than $n w$ nymphs, both at 15 days and 45 days posthatching (Table 1, Figure 1). The effect size of distance differences was considerable. 15-dayold $f w$ nymphs, which had a mean length of $3.81 \mathrm{~mm}$, moved an average of ca. $300 \mathrm{~mm}$ further than $n w$ nymphs. This difference in distance moved is ca. $79 \mathrm{x}$ their body length in a relatively short period of 5 minutes. For 45 day-old nymphs, the average movement differential was $132.73 \mathrm{~mm}$ (ca. $12.9 \mathrm{x}$ the mean body length of a 45 day-old nymph).

Table 1. Linear models examining the effects of genotype on total distance travelled in open field tests by 15 day-old (top) and 45 day-old (bottom) juveniles

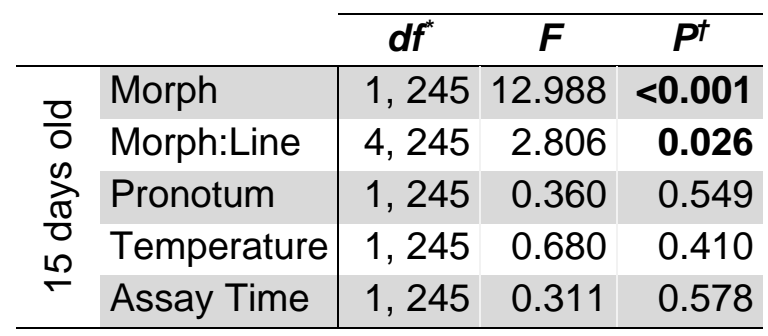

\begin{tabular}{|c|c|c|c|}
\hline \multirow{5}{*}{ 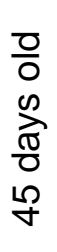 } & Morph & $1,21016.554$ & $<0.001$ \\
\hline & Morph:Line & $4,21012.696$ & $<0.001$ \\
\hline & Pronotum & $1,21012.122$ & $<0.001$ \\
\hline & Temperature & 1,210 & 0.455 \\
\hline & Assay Time & $1,21033.055$ & $<0.001$ \\
\hline
\end{tabular}

${ }^{*}$ numerator, denominator

${ }^{\dagger} \mathrm{p}<0.05$ indicated in bold 

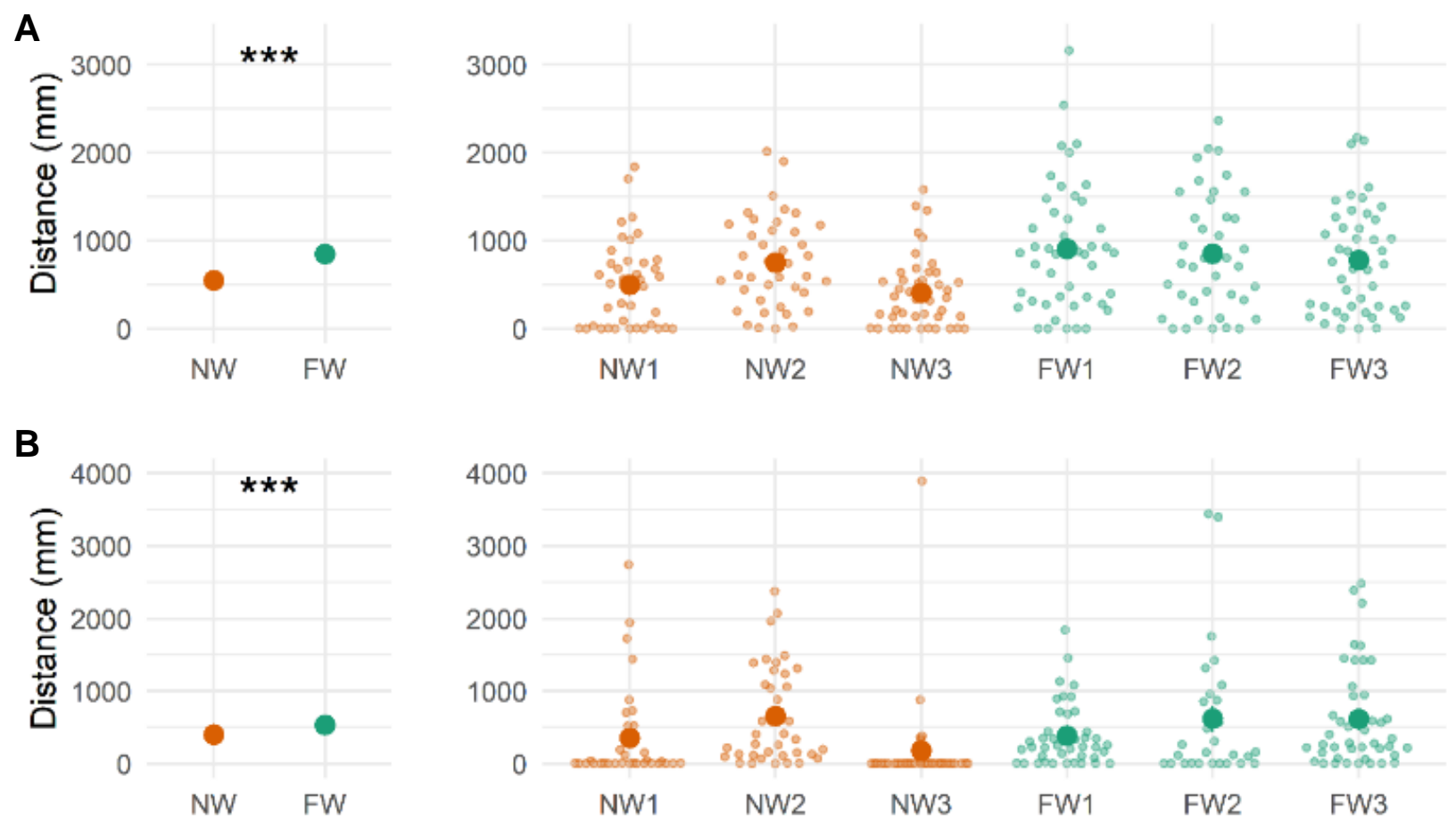

Figure 1. The effect of wing morph genotype on total distance traveled by juveniles in open field tests for (A) 15 day-old nymphs, (B) 45 day-old nymphs. Plots on the left illustrate pooled means across three replicate morph lines. Bars indicating \pm 1 standard error are not shown as these are too small to indicate graphically without being obstructed by the symbols for means. Violin plots on the right show data for each replicate morph line, with dark circles indicating means, small light circles showing each data point, and bars indicating \pm 1 standard error (when visible). ${ }^{* * *}$ indicates a morph difference with $p<0.001$. 


\section{Experiment 2: Social plasticity in the maternal generation (WGP)}

352 The acoustic social environment affected physiology (Table 2, Figure 2), but not mating

353 behavior (Supplementary Table S5; Supplementary Figure S4), in homozygous fw females.

354 Those raised in Song attained higher condition (SMI) (Table 2, Figure 2A) and had heavier

355 ovaries relative to somatic mass (Table 2, Figure 2B). Female mounting was not influenced

356 by prior acoustic experience, though females were more likely to mount higher condition

357 males (Supplementary Table S5 and Supplementary Figure S4A). Similarly, in trials where

358 females did mount males $(\mathrm{n}=49)$, there was no evidence that acoustic treatment affected

359 spermatophore transfer, though males were more likely to transfer a spermatophore to

360 higher condition females (Supplementary Table S5 and Supplementary Figure S4B).

Table 2. Linear models examining WGP arising from the acoustic environment on female condition (scaled mass index) and reproductive weight (g)

\begin{tabular}{l|c|c|c|ccc}
\cline { 2 - 7 } & \multicolumn{3}{c|}{ Condition } & \multicolumn{3}{c}{ Reproductive Weight } \\
\cline { 2 - 7 } & $\boldsymbol{d f}^{*}$ & $\boldsymbol{F}$ & $\boldsymbol{P}^{\boldsymbol{t}}$ & $\boldsymbol{d f}$ & $\boldsymbol{F}$ & $\boldsymbol{P}$ \\
\hline Acoustic treatment & 1,110 & 6.038 & $\mathbf{0 . 0 1 6}$ & 1,19 & 5.015 & $\mathbf{0 . 0 3 7}$ \\
Days isolated & 1,110 & 0.129 & 0.720 & 1,19 & 1.037 & 0.321 \\
Experimental replicate & 1,110 & 0.948 & 0.332 & - & - & - \\
\hline Somatic mass & - & - & - & 1,19 & 0.211 & 0.652 \\
\hline
\end{tabular}

* numerator, denominator

${ }^{\dagger} \mathrm{p}<0.05$ indicated in bold 

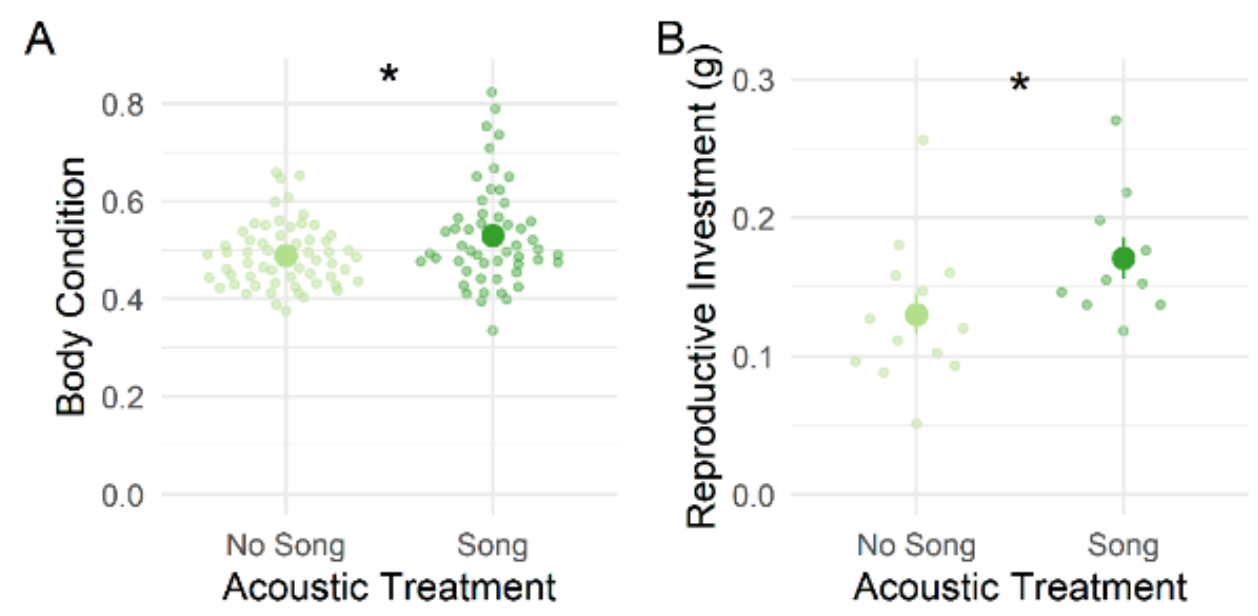

Figure 2. The effect of acoustic environment on (A) adult female body condition (scaled mass index) and (B) reproductive investment (ovaries mass). Plots show means \pm 1 standard error, except for (A), where standard error bars are too small to indicate graphically without being obstructed by the symbols for means. Asterisks indicate $p<0.05$. 

interactions between TGP and WGP in adult offspring

368 Unexpectedly, TGP affected adult, but not juvenile, traits (Figure 3). For example, the acoustic treatment of mothers was not associated with locomotion and morphology of their 15-day-old and 45-day old juvenile offspring (Table 4, Supplementary Tables S6 and S7).

371 However, adult offspring that experienced song themselves during rearing moved further

372 (WGP) (Table 5; Figure 3A), and in the case of males, this WGP was considerably exaggerated

373 if their mothers had been raised without song (TGP) (Figure 3A, right). It must be noted, this

374 WGP*TGP interaction was only marginally significant ( $p=0.055$; Table 5$)$, though the effect

375 size appears non-trivial (mean movement differential of ca. $400 \mathrm{~mm}$ for adult song-reared 376 males; Figure 3A, right).

Table 4. Linear models examining the effects of TGP arising from the maternal acoustic environment on total distance travelled by 15 day-old (top) and 45 day-old (bottom) juveniles

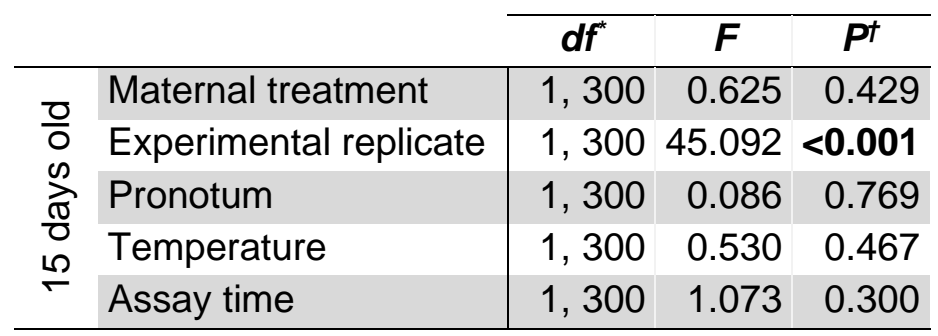

\begin{tabular}{|c|c|c|c|c|}
\hline 응 & Maternal treatment & 1,191 & 0.003 & 0.955 \\
\hline$\infty$ & Pronotum length & 1,191 & 5.714 & 0.017 \\
\hline$\frac{\pi}{0}$ & Temperature & 1,191 & 2.278 & 0.131 \\
\hline$\stackrel{1}{+}$ & Assay time & 1,191 & 0.338 & 0.561 \\
\hline
\end{tabular}

* numerator, denominator

$+p<0.05$ indicated in bold 

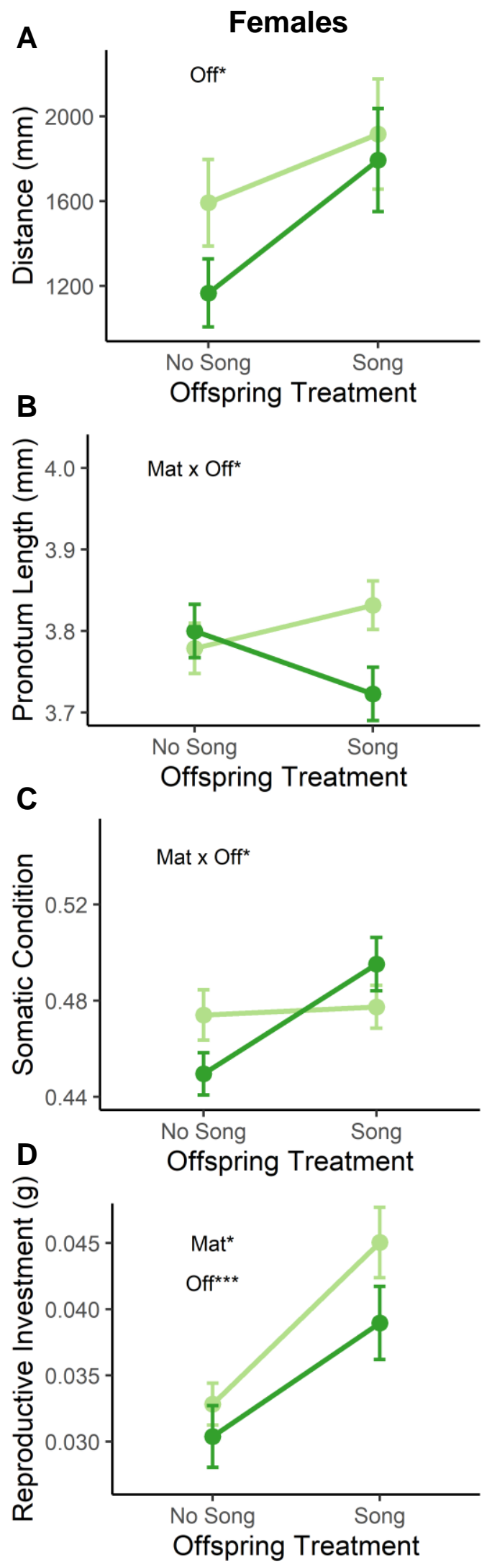

Maternal Treatment $=$ No Song $\gamma$ - Song

\section{Males}
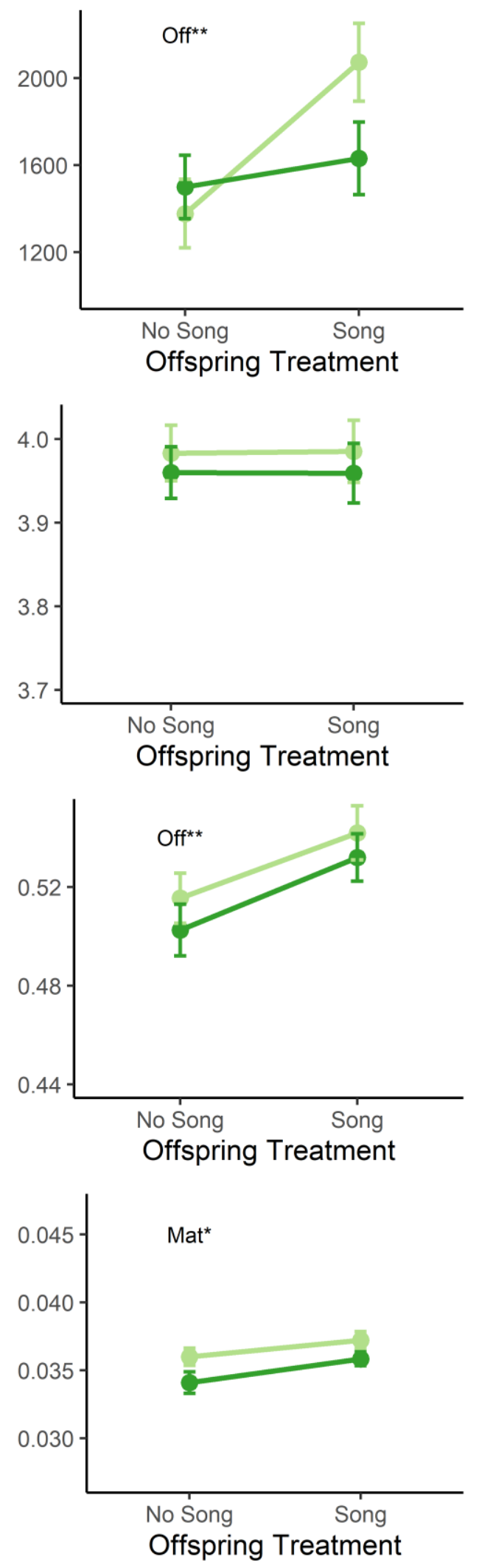

Figure 3. Effects of TGP and WGP in adult female (left) and adult male (right) offspring. (A) Distance travelled (B) pronotum length, $(\mathbf{C})$ somatic condition, (D) reproductive investment, i.e., female ovaries mass and male testis mass. Means are indicated by circles and bars indicate \pm 1 standard error. Significance of maternal ("Mat") treatment, offspring (“Off') treatment, and their interaction ("Mat x Off") are indicated with asterisks:

${ }^{*} p<0.05$

${ }^{* *} p<0.01$

${ }^{* * *} p<0.001$ 
Table 5. Linear models examining TGP and WGP on total distance moved by adult females (top) and adult males (bottom)

\begin{tabular}{|c|c|c|c|c|}
\hline & & $d f^{*}$ & $\boldsymbol{F}$ & $\overline{P t}$ \\
\hline \multirow{5}{*}{$\begin{array}{l}\mathscr{d} \\
\frac{\mathbb{d}}{\mathbb{D}} \\
\frac{\mathbb{d}}{\mathscr{D}}\end{array}$} & Maternal treatment & 1,181 & 2.055 & 0.152 \\
\hline & Offspring treatment & 1,181 & 6.279 & 0.012 \\
\hline & Temperature & 1,181 & 2.535 & 0.111 \\
\hline & Assay time & 1,181 & 1.923 & 0.166 \\
\hline & Somatic condition & 1,181 & 1.211 & 0.271 \\
\hline \multirow{6}{*}{$\frac{\mathscr{\Phi}}{\stackrel{\mathscr{N}}{\Sigma}}$} & Maternal treatment & 1,182 & 0.789 & 0.375 \\
\hline & Offspring treatment & 1,182 & 6.985 & 0.008 \\
\hline & Temperature & 1,182 & 6.620 & 0.010 \\
\hline & Time & 1,182 & 0.724 & 0.395 \\
\hline & Somatic condition & 1,182 & 3.567 & 0.059 \\
\hline & $\begin{array}{l}\text { Maternal treatment } \\
\text { Offspring treatment }\end{array}$ & 1,182 & 3.695 & 0.055 \\
\hline
\end{tabular}

* numerator, denominator

${ }^{\dagger} p<0.05$ indicated in bold

By contrast, acoustic effects on adult offspring morphology provided strong and consistent evidence for WGP, and TGP also affected several aspects of adult offspring morphology (Tables 6 and 7; Figure 3B,C,D). TGP often did not affect traits in the same direction as WGP; interactions between TGP and WGP combined to shape adult traits in a way that suggests TGP activates WGP, or put another way, that the manifestation of TGP is contingent on current environmental conditions. For example, female offspring reared without song had similar pronotum lengths, but when they were reared with song, those whose mothers experienced No Song grew to be larger than those whose mothers experienced Song (Table 6; Figure 3B, left). Female somatic condition showed a similar crossing-over effect (Table 6; Figure 3C, left). Also, TGP and WGP affected female investment in ovaries,

387 but in conflicting directions. Those raised in song developed heavier ovaries than those raised without song, and offspring from the No Song maternal treatment developed heavier ovaries than offspring from mothers who experienced Song (Table 7; Figure 3D, left). Adult males raised in the presence of song developed higher somatic condition than those raised without song, regardless of maternal treatment (Table 6; Figure 3B, right). We also found that crickets raised without song attempted flight more than those raised with song, particularly for females and lower condition individuals (Table 8; Figure 6; Supplementary Figure S5). 
Table 6. Linear models examining TGP and WGP on adult morphology for 394 females (top) and males (bottom)

\begin{tabular}{|c|c|c|c|c|c|c|c|}
\hline & & \multicolumn{3}{|c|}{ Pronotum Length } & \multicolumn{3}{|c|}{ Somatic Condition } \\
\hline & & $d f^{*}$ & $x^{2}$ & $P^{\dagger}$ & $d f$ & $x^{2}$ & $P$ \\
\hline \multirow{3}{*}{ 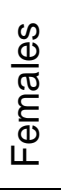 } & Maternal Treatment & 1,186 & 0.621 & 0.431 & 1,187 & 3.060 & 0.080 \\
\hline & Offspring Treatment & 1,186 & 1.452 & 0.228 & 1,187 & 0.079 & 0.779 \\
\hline & $\begin{array}{l}\text { Maternal Treatment* } \\
\text { Offspring Treatment }\end{array}$ & 1,186 & 4.741 & 0.029 & 1,187 & 4.670 & 0.031 \\
\hline \multirow{2}{*}{$\frac{\mathscr{\infty}}{\frac{\mathscr{N}}{\tilde{N}}}$} & Maternal Treatment & 1,187 & 0.199 & 0.655 & 1,187 & 1.265 & 0.261 \\
\hline & Offspring Treatment & 1,187 & 0.001 & 0.983 & 1,187 & 8.169 & 0.004 \\
\hline
\end{tabular}

Table 7. Linear models examining TGP and WGP on reproductive investment for adult females (top) and males (bottom)

\begin{tabular}{|c|c|c|c|c|}
\hline & \multirow[b]{2}{*}{$d f$} & \multirow{3}{*}{$\frac{x^{2}}{3.910}$} & \multirow{3}{*}{$\begin{array}{c}P \\
0.048\end{array}$} \\
\hline & & & & \\
\hline \multirow{4}{*}{$\begin{array}{l}\frac{\mathscr{D}}{\widetilde{J}} \\
\frac{\mathcal{D}}{\leftarrow} \\
\end{array}$} & Maternal treatment & 1,186 & & \\
\hline & Offspring treatment & 1,186 & 16.860 & $<0.001$ \\
\hline & Pronotum length & 1,186 & 0.894 & 0.345 \\
\hline & Somatic weight & 1,186 & 15.601 & $<0.001$ \\
\hline \multirow{4}{*}{$\frac{\mathscr{d}}{\sum^{\tilde{N}}}$} & Maternal treatment & 1,185 & 3.975 & 0.046 \\
\hline & Offspring treatment & 1,185 & 2.800 & 0.094 \\
\hline & Pronotum length & 1,185 & 1.698 & 0.193 \\
\hline & Somatic weight & 1,185 & 8.675 & 0.003 \\
\hline
\end{tabular}

* numerator, denominator

${ }^{\dagger} p<0.05$ indicated in bold

Table 8. GLMM examining the effects of WGP and TGP on flight attempts

\begin{tabular}{l|ccc}
\cline { 2 - 4 } \multicolumn{1}{c}{} & \multicolumn{1}{c}{$\boldsymbol{d f}$} & $\boldsymbol{X}^{2}$ & $\boldsymbol{P}$ \\
\hline Maternal treatment & 1,371 & 0.085 & 0.770 \\
\hline Offspring treatment & 1,371 & 8.788 & $\mathbf{0 . 0 0 3}$ \\
Sex & 1,371 & 4.213 & $\mathbf{0 . 0 4 0}$ \\
Somatic condition & 1,371 & 4.161 & $\mathbf{0 . 0 4 1}$ \\
Maternal treatment Sex & 1,371 & 2.140 & 0.144 \\
\hline${ }^{*}$ numerator, denominator \\
${ }^{\dagger} \mathrm{p}<0.05$ indicated in bold
\end{tabular}




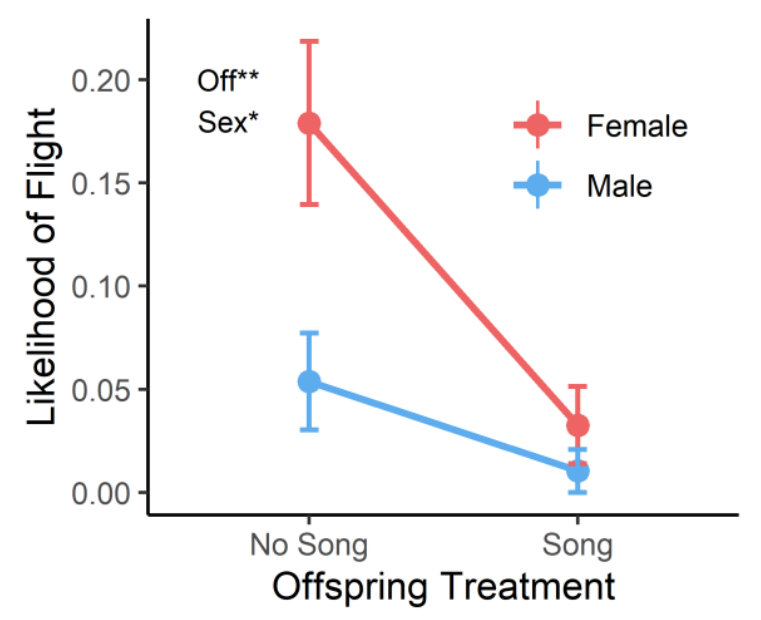

Figure 4. Likelihood of flight during open field trials. Means and \pm 1 standard error are represented by circles and bars, respectively. Significance of offspring ("Off") treatment and sex are indicated with asterisks: ${ }^{*} p<0.05,{ }^{* \star} p<0.01$.

\section{Discussion}

396 Phenotypic plasticity's role in evolution stimulates vigorous debate, but one barrier to a 397 general resolution may be that plasticity is not a monolithic phenomenon. Influential verbal 398 models have suggested that "buffering" effects of plasticity can permit novel adaptations to 399 escape loss at low frequencies and subsequently spread under selection, but few empirical 400 studies have been able to assess the contributions and interactions of genetics and different 401 forms of phenotypic plasticity such as within-generation and transgenerational plasticity. 402 Here we demonstrate how all three inputs - genetics, WGP and TGP - interact to affect traits 403 that potentially facilitate the rapid evolution of a parasitoid-avoidance adaptation in 404 Hawaiian field crickets, male silence.

Genotype had a surprisingly large effect on juvenile behavior, but in the opposite 406 direction predicted. At very early juvenile stages, flatwing carriers moved nearly 80 body 407 lengths further than normal-wing carriers in a span of only 5 minutes. This genotypic 408 difference could result from pleiotropic effects of the fw mutation or genomic hitchhiking. 409 Genotypic differences in juvenile locomotion are consistent with phenotypic differences that 410 have been detected between $f s$ and $n w$ carriers in other sexually dimorphic adult traits 
411 (Pascoal et al. 2016; Rayner et al. 2019b; Pascoal et al. 2020), which raises the possibility 412 that the $f w$ genotype may be exposed to selection at an earlier stage than previously 413 considered, for example through associated effects on foraging efficiency or predation risk.

414 Further, it is possible that increased locomotion of $f w$ juveniles might have accelerated the speed at which the mutation initially spread in the wild. Rather than facilitating local mating

416 aggregations as we initially hypothesized, greater movement activity may instead permit

417 silent crickets and females carrying male-silencing variants to encounter one another. The

418 ultimate fitness consequences of these differences remain to be tested, but more broadly, this

419 result illustrates how genetic correlations manifesting during development might impact the

420 trajectory of a mutant genotype which carries fitness benefits at adult stages. The idea that

421 advantageous mutations can have pleiotropic effects during development has been explored

422 extensively in the context of insecticide resistance and alternative reproductive morphs

423 (Boivin et al. 2001; Giraldo-Deck et al. 2020). Against the background of this work, our

424 findings suggest the combined phenotypic and fitness effects of adaptive mutations arising 425 from positive pleiotropy, negative pleiotropy, and genomic hitchhiking may be non-intuitive, 426 and phenotypic variation caused by such effects can alter the dynamics of adaptive evolution 427 as well as the manner in which plasticity affects that evolution.

In the crickets, transgenerational plasticity and within-generation plasticity sometimes acted in concert, sometimes in opposition, and in some cases did not appear influential in shaping trait variation. If TGP is adaptive for offspring (and therefore also for mothers), a match between parental and offspring environment should result in higher 432 offspring fitness when compared to mis-matched offspring (Marshall and Uller 2007; Uller et 433 al. 2013). However, we found no evidence supporting this expectation for offspring performance traits. Patterns of reproductive investment provide an illustrative example. Consistent with previous studies in this system and in other cricket species (Bailey et al. 2010; Conroy and Roff 2018), mothers who experienced No Song invested less in reproductive tissue. Such WGP is in line with adaptive predictions, as it allows individuals to reallocate resources to non-reproductive tissue when competition and/or opportunity for mating is low 440 dominated social environment where no males can sing, because plasticity shifting resources 441 from reproduction to survival could increase the chances that a female survives long enough 442 to find a mate. Consistent effects of TGP and WGP on offspring would facilitate this, but 
443 instead we found that TGP and offspring WGP acted in opposing directions (Figure 3D). It is

444 therefore unlikely that this TGP in reproductive investment is an adaptive, anticipatory

445 effect to increase offspring fitness, but instead may be an incidentally-transmitted physiological consequence of mothers responding to their social environment ("selfish TGP" cf. Marshall and Uller 2007) or cross-generation spillover of parental condition ("conditiontransfer effects" cf. Bonduriansky and Crean 2018). Lack of support for adaptive TGP is consistent with a recent meta-analysis which recovered weak evidence for it across taxa 450 (Uller et al. 2013).

WGP has been suggested to be more efficient than TGP, so once capable of assessing their environment, offspring are expected to rewrite parental cues with their own (Ezard et al. 2014; Auge et al. 2017; Moore et al. 2019). Nevertheless, we found that the maternal social environment affected adult, but not juvenile, offspring phenotype in T. oceanicus. One reason for the delayed action of TGP might be that the maternal social environment influenced phenotype via mechanisms that would not cause observable differences until late in development. For example, many insects exhibit significant plasticity in the number of instars they undergo prior to sexual maturation, which can affect sexual size dimorphism at adulthood (Esperk et al. 2007; Stillwell et al. 2010). Another possibility is that the maternal social environment influenced offspring phenotype very early in development, such as size at hatching, but those effects dissipated prior to later phenotypic measurement, as was found in the salamander Ambystoma talpoideum (Moore et al. 2015). A third possibility is that TGP mediated by maternal social environments could be qualitatively different from TGP mediated by maternal physical environments. For example, nutritional or thermal environments that mothers experience may have more direct impacts on juvenile offspring, whereas the social environment comprised of adult social cues is likely to be of greater relevance to offspring when they are adults themselves.

470 TGP. This supports the prediction that traits whose expression remains flexible after 471 development are more strongly affected by WGP (Beaty et al. 2016). Specifically, offspring of 472 both sexes were more active when reared in song. They may increase walking activity to 473 locate conspecifics they perceive to be abundant nearby, even in the absence of an immediate 474 acoustic cue. In contrast, crickets raised without song have no indication of nearby 
475

476

477

478

479

480

481

482

483

484

485

486

487

488

489

490

491

492

493

494

495

496

497

498

499

500

501

502

503

504

505

506

conspecifics and may decrease short-range mate-searching via walking to instead wait for an acoustic cue. This trade-off is likely motivated by a high metabolic cost of mate-searching (Hack 1998) and the resulting increase in predation risk (Bell 1990). Our results contrast with previous studies in this species which found that adult males raised in song are less active than those raised in silence (Balenger and Zuk 2015) and that females exhibit limited flexibility in locomotive behavior in response to acoustic environment (Heinen-Kay et al. 2018), but are consistent with other findings that suggest increases in exploratory behavior under predation risk (Moschilla et al. 2021).

One explanation for this apparent inconsistency is that the latter studies conducted movement trials in environments that contained shelter or cover and in some cases assessed movement towards acoustic stimuli, whereas we used an open field test to mimic the experience of Hawaiian crickets within an all-flatwing population, in which locomotion in the absence of any immediately available acoustic signals is likely to have significant fitness consequences. Cover during movement trials could have reduced the perception of risk associated with walking, making increased undirected mate-searching in a song-less environment advantageous (Hedrick and Dill 1993). Additionally, two of the previous studies relied on indirect measures of activity (e.g., time spent walking, gridlines crossed, farthest grid reached), whereas we directly measured distance traveled using automated and validated coordinate collection, giving us greater resolution to resolve variation in activity. Finally, we made an incidental discovery during the course of the experiment which provides another intriguing explanation: offspring reared in No Song were more likely to attempt flight during the open field test, a pattern that was particularly strong in females who were ca. 4 times more likely to attempt fly if they had experienced no acoustic signals during development (Figure 4). Individuals reared without song may be more disposed to use flight as part of an un-directed, long-range dispersal strategy akin to Lévy flight (Viswanathan et al. 1996) to increase their chances of reaching an area of greater conspecific resources. Trading off increased long-range dispersal via flight with decreased walking behavior in song-less, flatwing-dominated environments could have increased the speed at which flatwing alleles spread under pressure from parasitoid flies.

Our results support the idea that the effects of TGP can be contingent upon offspring environment. Put another way, sometimes TGP potentiates WGP, and sometimes it suppresses WGP. It is therefore necessary to consider the potentially conflicting effects of 
WGP and TGP when predicting how phenotypic plasticity influences adaptive evolution. Theory predicts that, following rapid environmental change, populations may exhibit a transient increase in plasticity because genotypes which shift trait expression closer to a new optimum are favored (Lande 2009). Additionally, genotypes coding for reaction norm slopes of other traits that offset negative effects of new variants spreading under selection may also be favored, as appears to be the case in T. oceanicus in Hawaii and other systems (Bailey et al. 2021). If increases in WGP during an adaptive evolutionary response result in a spillover of non-adaptive TGP to the offspring generation, the role of plasticity in facilitating the establishment and spread of novel adaptations may be less straightforward than currently understood (Lacey 1998; Bonduriansky and Day 2009; Bell and Hellmann 2019). Previous work in the Hawaiian flatwing cricket system supports predictions of increased WGP which mitigates negative pleiotropy of male silence, in this case facilitating mate location and reproduction in a song-less social environment (Bailey and Zuk 2008; Bailey et al. 2018; Tinghitella et al. 2009; Bailey 2011; Bailey and Zuk 2012; Balenger and Zuk 2015; Pascoal et al. 2018), but see (Rayner et al. 2020). However, we did not find a prominent signature of either adaptive TGP or of an adaptive interaction between TGP and WGP. Instead, our results suggest that plasticity transmitted across generations may have more complex effects and, in some cases, counterbalance facilitating effects of WGP.

\section{References}

Allen RM, Buckley YM, Marshall DJ. 2008. Offspring size plasticity in response to intraspecific competition: An adaptive maternal effect across life-history stages. Am Nat. 171(2):225-237. doi:10.1086/524952.

Auge GA, Leverett LD, Edwards BR, Donohue K. 2017. Adjusting phenotypes via within- and acrossgenerational plasticity. New Phytol. 216(2):343-349. doi:10.1111/nph.14495.

Bailey NW. 2011. Mate choice plasticity in the field cricket Teleogryllus oceanicus: Effects of social experience in multiple modalities. Behav Ecol Sociobiol. 65(12):2269-2278. doi:10.1007/s00265-0111237-8.

Bailey NW, Desjonquères C, Drago A, Rayner JG, Sturiale SL, Zhang X. 2021. A neglected conceptual problem regarding phenotypic plasticity's role in adaptive evolution: The importance of genetic covariance and social drive. Evol Lett.:1-14. doi:10.1002/evl3.251.

Bailey NW, Gray B, Zuk M. 2010. Acoustic Experience Shapes Alternative Mating Tactics and 

the field cricket Teleogryllus oceanicus. Behav Ecol 19:202-207. doi:10.1093/beheco/arm123.

542 Bailey NW, Zuk M. 2008. Acoustic experience shapes female mate choice in field crickets. Proc R Soc 543 B Biol Sci. 275(1651):2645-2650. doi:10.1098/rspb.2008.0859.

544 Bailey NW, Zuk M. 2012. Socially flexible female choice differs among populations of the Pacific field 545 cricket: geographical variation in the interaction coefficient psi (). Proc R Soc B Biol Sci.

546 279(May):3589-3596. doi:10.1098/rspb.2012.0631.

547 Balenger SL, Zuk M. 2015. Roaming Romeos: Male crickets evolving in silence show increased

548 locomotor behaviours. Anim Behav. 101:213-219. doi:10.1016/j.anbehav.2014.12.023.

549 http://dx.doi.org/10.1016/j.anbehav.2014.12.023.

550 Beaty LE, Wormington JD, Kensinger BJ, Bayley KN, Goeppner SR, Gustafson KD, Luttbeg B.

551 2016. Shaped by the past, acting in the present: transgenerational plasticity of anti-predatory traits.

552 Oikos. 125(11):1570-1576. doi:10.1111/oik.03114.

553 Bell AM, Hellmann J. 2019. An Integrative Framework for Understanding the Mechanisms and

554 Multigenerational Consequences of Transgenerational Plasticity. Annu Rev Ecol Evol Syst. 50(1):97555 118. doi:10.1146/annurev-ecolsys-110218-024613.

556 Bell WJ. 1990. Searching Behavior Patterns In Insects. Annu Rev Entomol. 35:447-467.

557 doi:https://doi.org/10.1146/annurev.en.35.010190.002311.

558 Boivin T, Chabert D’Hières C, Bouvier JC, Beslay D, Sauphanor B. 2001. Pleiotropy of insecticide

559 resistance in the codling moth, Cydia pomonella. Entomol Exp Appl. 99(3):381-386.

560 doi:10.1046/j.1570-7458.2001.00838.x.

561 Bonduriansky R, Crean A. 2018. What are parental condition-transfer effects and how can they be 562 detected? Methods Ecol Evol. 9(3):450-456. doi:10.1111/2041-210X.12848.

563 Bonduriansky R, Day T. 2009. Nongenetic inheritance and its evolutionary implications. Annu Rev 564 Ecol Evol Syst. 40:103-125. doi:10.1146/annurev.ecolsys.39.110707.173441.

565 Chevin LM, Lande R, Mace GM. 2010. Adaptation, plasticity, and extinction in a changing 566 environment: Towards a predictive theory. PLoS Biol. 8(4). doi:10.1371/journal.pbio.1000357.

567 Conroy LP, Roff DA. 2018. Adult social environment alters female reproductive investment in the 
cricket Gryllus firmus. Behav Ecol. 29(2):440-447. doi:10.1093/beheco/arx193.

569 Denno RF, Roderick GK. 1992. Density-Related Dispersal in Planthoppers : Effects of Interspecific

570 Crowding. Ecology. 73(4):1323-1334. doi:https://doi.org/10.2307/1940679.

571 Dingemanse NJ, Both C, Van Noordwijk AJ, Rutten AL, Drent PJ. 2003. Natal dispersal and

572 personalities in great tits (Parus major). Proc R Soc B Biol Sci. 270(1516):741-747.

573 doi:10.1098/rspb.2002.2300.

574 Dyer AR, Brown CS, Espeland EK, McKay JK, Meimberg H, Rice KJ. 2010. The role of adaptive

575 trans-generational plasticity in biological invasions of plants. Evol Appl. 3(2):179-192.

576 doi:10.1111/j.1752-4571.2010.00118.x.

577 Esperk T, Tammaru T, Nylin S, Teder T. 2007. Achieving high sexual size dimorphism in insects:

578 Females add instars. Ecol Entomol. 32(3):243-256. doi:10.1111/j.1365-2311.2007.00872.x.

579 Ezard THG, Prizak R, Hoyle RB. 2014. The fitness costs of adaptation via phenotypic plasticity and 580 maternal effects. Funct Ecol. 28(3):693-701. doi:10.1111/1365-2435.12207.

581 Fraser DF, Gilliam JF, Daley MJ, Le AN, Skalski GT. 2001. Explaining leptokurtic movement

582 distributions: Intrapopulation variation in boldness and exploration. Am Nat. 158(2):124-135.

583 doi:10.1086/321307.

584 Friard OF. 2019. DORIS.

585 Ghalambor CK, McKay JK, Carroll SP, Reznick DN. 2007. Adaptive versus non-adaptive phenotypic 586 plasticity and the potential for contemporary adaptation in new environments. Funct Ecol.

587 21(3):394-407. doi:10.1111/j.1365-2435.2007.01283.x.

588 Giraldo-Deck LM, Goymann W, Safari I, Dawson DA, Stocks M, Burke T, Lank DB, Küpper C. 2020.

589 Development of intraspecific size variation in black coucals, white-browed coucals and ruffs from

590 hatching to fledging. J Avian Biol. 51(8):1-14. doi:10.1111/jav.02440.

591 Hack MA. 1998. The energetics of male mating strategies in field crickets (Orthoptera: Gryllinae:

592 Gryllidae). J Insect Behav. 11(6):853-867. doi:10.1023/A:1020864111073.

593 Harshman LG, Zera AJ. 2007. The cost of reproduction: the devil in the details. Trends Ecol Evol. 594 22(2):80-86. doi:10.1016/j.tree.2006.10.008.

595 Hedrick A V., Dill LM. 1993. Mate choice by female crickets is influenced by predation risk. Anim 596 Behav. 46:193-196. doi:10.1006/anbe.1993.1176.

597 Heinen-Kay JL, Strub DB, Balenger SL, Zuk M. 2019. Direct and indirect effects of sexual signal loss 
on female reproduction in the Pacific field cricket (Teleogryllus oceanicus). J Evol Biol.(May):1-9. doi:10.1111/jeb.13534.

Heinen-Kay JL, Strub DB, Zuk M. 2018. Limited flexibility in female Pacific field cricket (Teleogryllus oceanicus) exploratory behaviors in response to perceived social environment. Ethology. 124(9):650-656. doi:10.1111/eth.12767.

Huey RB, Hertz PE, Sinervo B. 2003. Behavioral drive versus behavioral inertia in evolution: A null model approach. Am Nat. 161(3):357-366. doi:10.1086/346135.

Korsten P, Van Overveld T, Adriaensen F, Matthysen E. 2013. Genetic integration of local dispersal and exploratory behaviour in a wild bird. Nat Commun. 4(2362). doi:10.1038/ncomms3362.

Lacey ER. 1998. What Is an Adaptive Environmentally Induced Parental Effect? Oxford Univ Press.:54-66.

LaMontagne JM, McCauley E. 2001. Maternal effects in Daphnia: What mothers are telling their offspring and do they listen? Ecol Lett. 4(1):64-71. doi:10.1046/j.1461-0248.2001.00197.x.

Lande R. 2009. Adaptation to an extraordinary environment by evolution of phenotypic plasticity and genetic assimilation. J Evol Biol. 22(7):1435-1446. doi:10.1111/j.1420-9101.2009.01754.x.

Leimar O, McNamara JM. 2015. The evolution of transgenerational integration of information in heterogeneous environments. Am Nat. 185(3):55-69. doi:10.1086/679575.

Lierheimer VF, Tinghitella RM. 2017. Quantity and quality of available mates alters female responsiveness but not investment in the Pacific field cricket, Teleogryllus oceanicus. Behav Ecol Sociobiol. 71(5). doi:10.1007/s00265-017-2298-0.

Marshall DJ, Uller T. 2007. When is a maternal effect adaptive? Oikos. 116(12):1957-1963. doi:10.1111/j.2007.0030-1299.16203.x.

Moore MP, Landberg T, Whiteman HH. 2015. Maternal investment mediates offspring life history variation with context-dependent fitness consequences. Ecology. 96(9):2499-2509. doi:10.1890/141602.1.

Moore MP, Whiteman HH, Martin RA. 2019. A mother's legacy: the strength of maternal effects in animal populations. Ecol Lett. 22(10):1620-1628. doi:10.1111/ele.13351.

Moschilla JA, Tomkins JL, Simmons LW. 2021. Nongenetic inheritance of behavioural variability is context specific and sex specific. Funct Ecol. doi:10.1111/1365-2435.13931.

Pascoal S, Liu X, Fang Y, Paterson S, Ritchie MG, Rockliffe N, Zuk M, Bailey NW. 2018. Increased 
socially mediated plasticity in gene expression accompanies rapid adaptive evolution. Ecol Lett. 21(4):546-556. doi:10.1111/ele.12920.

Pascoal S, Liu X, Ly T, Fang Y, Rockliffe N, Paterson S, Shirran SL, Botting CH, Bailey NW. 2016. Rapid evolution and gene expression: a rapidly evolving Mendelian trait that silences field crickets has widespread effects on mRNA and protein expression. J Evol Biol. 29(6):1234-1246. doi:10.1111/jeb.12865.

Pascoal S, Risse JE, Zhang X, Blaxter M, Cezard T, Challis RJ, Gharbi K, Hunt J, Kumar S, Langan E, et al. 2020. Field cricket genome reveals the footprint of recent, abrupt adaptation in the wild. Evol Lett. 4(1):19-33. doi:10.1002/evl3.148.

R Core Team. 2020. R: A Language and Environment for Statistical Computing. https://www.rproject.org/.

Rayner JG, Aldridge S, Montealegre-Z F, Bailey NW. 2019a. A silent orchestra: convergent song loss in Hawaiian crickets is repeated, morphologically varied, and widespread. Ecology. 100(8):1-4. doi:10.1002/ecy.2694.

Rayner JG, Pascoal S, Bailey NW. 2019b. Release from intralocus sexual conflict? Evolved loss of a male sexual trait demasculinizes female gene expression. Proc R Soc B Biol Sci. 286(1901). doi:10.1098/rspb.2019.0497.

Rayner JG, Schneider WT, Bailey NW. 2020. Can behaviour impede evolution? Persistence of singing effort after morphological song loss in crickets: Singing effort in mute crickets. Biol Lett. 16(6). doi:10.1098/rsbl.2019.0931.

Robinson BW, Dukas R. 1999. The Influence of Phenotypic Modifications on Evolution : The Baldwin Effect and Modern Perspectives. Oikos. 85(3):582-589. doi:https://doi.org/10.2307/3546709.

Sheriff MJ, Krebs CJ, Boonstra R. 2010. The ghosts of predators past: Population cycles and the role of maternal programming under fluctuating predation risk. Ecology. 91(10):2983-2994. doi:10.1890/09-1108.1.

Staudacher EM. 2009. The auditory system of last instars in Gryllus bimaculatus DeGeer. Physiol Entomol. 34(1):18-29. doi:10.1111/j.1365-3032.2008.00647.x.

Stillwell RC, Blanckenhorn WU, Teder T, Davidowitz G, Fox CW. 2010. Sex Differences in Phenotypic Plasticity Affect Variation in Sexual Size Dimorphism in Insects: From Physiology to Evolution. Annu Rev Entomol. 55(1):227-245. doi:10.1146/annurev-ento-112408-085500.

Swanger E, Zuk M. 2015. Cricket Responses to Sexual Signals are Influenced More by Adult than 
Juvenile Experiences. J Insect Behav. 28(3):328-337. doi:10.1007/s10905-015-9504-6.

660 Tinghitella RM, Broder ED, Gurule-Small GA, Hallagan CJ, Wilson JD. 2018. Purring crickets: The

661 evolution of a novel sexual signal. Am Nat. 192(6):773-782. doi:10.1086/700116.

662 Tinghitella RM, Wang JM, Zuk M. 2009. Preexisting behavior renders a mutation adaptive:

663 Flexibility in male phonotaxis behavior and the loss of singing ability in the field cricket Teleogryllus

664 oceanicus. Behav Ecol. 20(4):722-728. doi:10.1093/beheco/arp052.

665 Tomkins JL, Simmons LW. 2002. Measuring relative investment: A case study of testes investment

666 in species with alternative male reproductive tactics. Anim Behav. 63(5):1009-1016.

667 doi:10.1006/anbe.2001.1994.

668 Uller T, Nakagawa S, English S. 2013. Weak evidence for anticipatory parental effects in plants and 669 animals. J Evol Biol. 26(10):2161-2170. doi:10.1111/jeb.12212.

670 Viswanathan GM, Afanasyev V, Buldyrev S V., Murphy EJ, Prince PA, Stanley HE. 1996. Lévy

671 flight search patterns of wandering albatrosses. Nature. 381(6581):413-415. doi:10.1038/381413a0.

672 West-Eberhard MJ. 2003. Developmental plasticity and evolution. Nature. 424:794.

673 doi:10.2002/ajpa.20219.

674 Yack JE. 2004. The structure and function of auditory chordotonal organs in insects. Microsc Res

675 Tech. 63(6):315-337. doi:10.1002/jemt.20051.

676 Young D, Ball E. 1974. Structure and development of the auditory system in the prothoracic leg of 677 the cricket Teleogryllus commodus (walker) - I. Adult structure. Zeitschrift für Zellforsch und 678 mikroskopische Anat. 147(3):293-312. doi:10.1007/BF00307466.

679 Yu EY, Gassmann AJ, Sappington TW. 2019. Effects of larval density on dispersal and fecundity of 680 western corn rootworm, Diabrotica virgifera virgifera LeConte (Coleoptera: Chrysomelidae). PLoS

681 One. 14(3):1-19. doi:10.1371/journal.pone.0212696.

682 Zuk M, Rotenberry JT, Tinghitella RM. 2006. Silent night: Adaptive disappearance of a sexual signal 683 in a parasitized population of field crickets. Biol Lett. 2(4):521-524. doi:10.1098/rsbl.2006.0539.

684 Zuk M, Simmons LW, Cupp L. 1993. Calling Characteristics of Parasitized and Unparasitized 685 Populations of the Field Cricket Teleogryllus oceanicus. Behav Ecol Sociobiol. 33(5):339-343. 686 doi:https://doi.org/10.1007/BF00172933. 